\title{
On Failure Modes and Strength Characterization of Brittle Disordered Materials under Uniaxial Compression and Tension
}

\author{
C.A. Tang ${ }^{1,2}$, P. Lin ${ }^{1}$, H.Y. Liu ${ }^{1}$ and Z.Z. Liang ${ }^{1}$ \\ ${ }^{1}$ CRISR, Resources and Civil Engineering School, Northeastern University, \\ Shenyang 110006, China P.R. \\ ${ }^{2}$ LNM, Institute of Mechanics, Chinese Academy of Sciences, \\ Beijing 100080, China P.R.
}

Keywords: Brittle Failure, Heterogeneity, Localization, Nonlinear Behavior, Numerical Simulation, Strength

\begin{abstract}
With a newly developed Material Failure Process Analysis code $\left(\mathrm{MFPA}^{2 \mathrm{D}}\right)$, influence of hetero geneity on fracture processes and strength characterization of brittle disorder materials such as rock or concrete is numerically studied under uniaxial compression and tension conditions. It is found th at, due to the heterogeneity of the disordered material, relatively more diffused micro-fractures appe ar in the early stage of loading. Different from homogeneous materials such as glass, macro-crack n ucleation starts well before the peak stress is reached and the crack propagation and coalescence can be traced, which can be taken as a precursory to predict the macro-fracture of the material. The pres ence of residual strength in the post-peak region and the resemblance in the stress-strain curves bet ween tension and compression are significant results and are found to be dependent on the heteroge neity of the specimens. Examples showing the tentative applications of $\mathrm{MFPA}^{2 \mathrm{D}}$ in modeling failure of composite materials and rock or civil engineering problem are also given in this paper.
\end{abstract}

\section{INTRODUCTION}

The failure behavior of quasi-brittle materials such as rock or concrete is characterized by nonlin ear deformation prior to the peak strength is attained, followed by the weakening (or sometimes call ed softening [1] ) and localization of deformation in the post-peak part. Thus, neither linear elastic $f$ racture mechanics nor plasticity models can be adequately used to describe such behavior [2]. Altho ugh nonlinear fracture mechanics or nonlocal continuum models can provide a general description, $t$ hey are incapable of capturing the influence of the heterogeneity at the micro or meso-level [2] and therefore difficult to be used to investigate the micro-fracture induced progressive failure of the diso rdered materials. Even under uniform loading conditions, the stress distribution in micro or meso sc ale is not uniform since rock or concrete materials contain numerous small defects that are randoml $\mathrm{y}$ distributed within the materials. These defects significantly affect the physical properties of the $\mathrm{m}$ aterials and result in local disorder features of the stress distribution if loaded. Consequently, direct simulation of the randomness of disordered material microstructure is useful in arriving at a better $u$ nderstanding of brittle failure and the improved prediction of the mechanical properties.

Based on experimental observations, material behavior models can be constructed. The models $c$ an be either analytical or numerical. The analytical models lead to correct answers within the frame 
work of axioms underlying the mathematics. However, in most of the cases, analytical models have to be simplified and sometimes this simplification ignores very important factors influencing the ma terial behavior. Heterogeneity is such an example for rock or concrete.

Statistical modeling has emerged as a promising technique for analysis of fracture in heterogene ous materials such as rock or concrete [3]. The combinations of statistical theory with numerical mo dels such as the lattice model [4], the bonded particle model [5-6], or tensile fracture models based on FEM [2.7] are found to be quite appropriate for modeling brittle disorder materials such as rocks

In this paper, uniaxial tensile and compressive tests with specimens of brittle disordered materi als were numerically studied by using a Material Failure Process Analysis code (MFPA ${ }^{2 \mathrm{D}}$ ), develope d recently by CRISR at Northeastern University [1]. Following a brief description of the numerical model and the loading procedures adopted in this investigation, some characteristic features of the $\mathrm{c}$ omplete stress-strain curves under uniaxial tension and compression and the phenomena observed $d$ uring progressive fracture of disordered materials will be summarized in terms of heterogeneity, def ormation localization, fracture nucleation or coalescence, and micro-fracture induced seismic activit ies. Finally, some examples showing the tentative applications of $\mathrm{MFPA}^{2 \mathrm{D}}$ in modeling failure of co mposite materials and rock or civil engineering problems are also given in this paper.

\section{BRIEF OUTLINE OF MFPA ${ }^{2 D}$}

Numerical simulation is currently the most popular method used for modeling deformation beha vior of brittle materials before failure. Even though progress has been made in numerical simulation of failure occurring in these materials, there is a lack of satisfactory models that can simulate the pr ogressive failure in a more visualized way, including simulation of the failure process, failure induc ed seismic events and failure induced stress redistribution.

The demand for new tools, which may contribute to a better understanding of the failure mechanisms of brittle materials, has initiated the development of MFPA $^{2 D}$ (Material Failure Process Analysis code) based on RFPA ${ }^{2 D}$. The code, developed at CRISR, Northeastern University, China, can be used to model failure process of rock by considering the deformation of an elastic material containing a randomly initial distribution of micro-defects. Details of the model are given in [1], so only a few important points will be presented here.

Briefly, the disordered material properties (failure-strength $\sigma_{c}$ and elastic module $e_{c}$ ) for elements are randomly distributed throughout the specimen by following a Weibull's distribution. We use a smeared failure approach that a micro-fracturing occurs when the stress of an element satisfies a strength criterion. A Coulomb criterion envelope with a tensile cut-off is used so that the failure of the elements may be either in shear or in tensile.

MFPA $^{2 D}$ is a Microsoft Windows application package for material failure analysis of mines/geote chnical structures using finite element technique as a calculator. A user-friendly pre- and post-proce ssor is integrated to generate the model mesh and prepare the data input. The result may be displaye $\mathrm{d}$ as an "animation" to aid the understanding of the mechanics of collapse.

\section{MODEL PREDICTIONS FOR COMPRESSIVE AND DIRECT TENSILE LOADING}

\subsection{Influence of Heterogeneity on Stress-Strain Response and Strength Characterization}

The heterogeneity of disordered materials has an important influence on the shape of stress-strain curves and the strength characterization. Fig.1 shows the simulated stress-strain curves in 
compression for specimens with various homogeneity indices, $m=1.5,2.3$, and 5 (the smaller the value $m$, the wider the strength distribution). The specimens have a geometry of $150 \times 100 \mathrm{~mm}$. The load is applied in the vertical direction through displacement control. The simulations show that wider strength distributions yield an earlier onset of non-linear behavior and lower pear stresses, but a more gradual loss of strength after the peak.

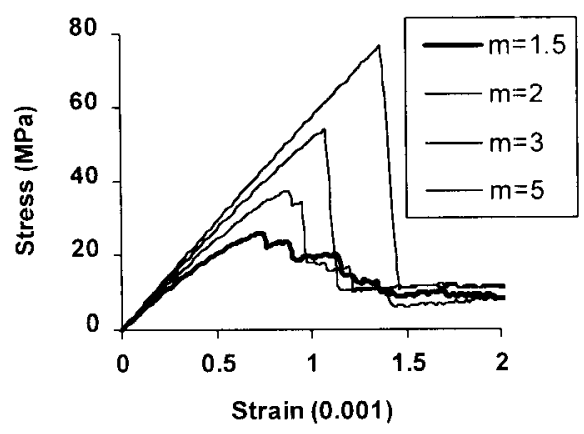

Fig.1 Influence of heterogeneity on stressStrain curves in compression

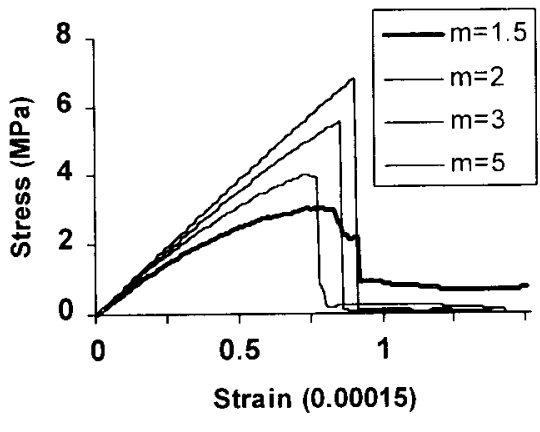

Fig.2 Influence of heterogeneity on stressstrain curves in tension

It is generally considered that the microscopic failure mechanisms in tension and compression ar e considerably different from each other. In compression, a failed portion does not completely lose $\mathrm{i}$ ts loading bearing capacity because of friction with the surroundings. However, in tension the loadi ng capacity of failed portion decreases more rapidly than in compression. This consideration sugges ts a relative sharp decrease of loading bearing capacity of a specimen in tension. However, as obser ved by Okubo and Fukui [8] in their experiments. the shapes of the stress-strain curves do not differ much from each other in tension and compression. This has also been verified with our numerical s imulations. Fig. 2 shows the simulated stress-strain curves in tension for specimens with the same ho mogeneity indices as in compression. The resemblance in the stress-strain curves between tension a nd compression can be easily seen by comparing this Fig. 2 with Fig.1. Though exact explanations $f$ or this result are difficult, heterogeneity mentioned above at least can be taken as one of the reasons for this resemblance.

Simulations of both compressive and tensile tests reveal that the maximum strength of the specimens is proportional to the homogeneity index.

\subsection{Influence of Heterogeneity on Microfracture Behavior}

The numerical results also demonstrate that the $\mathrm{AE}$ (acoustic emissions) event patterns are influenced greatly by the degree of heterogeneity of the materials. Fig. 3 shows that AE count rate as a function of deformation for the four specimens with different homogeneity index. A comparison between Fig. 1 and Fig. 3 shows a good relationship between the modeled stress-strain curves and the modeled curves of event rate. The results show that the relatively heterogeneous specimen emits more $\mathrm{AE}$ events as the precursory of macro-fracture than that of the relatively homogeneous specimen. On the other hand, higher stress drop is observed to be correspondent to the higher event rate in relatively homogeneous specimens. The same conclusion may also be obtained from the simulation of tension tests. 

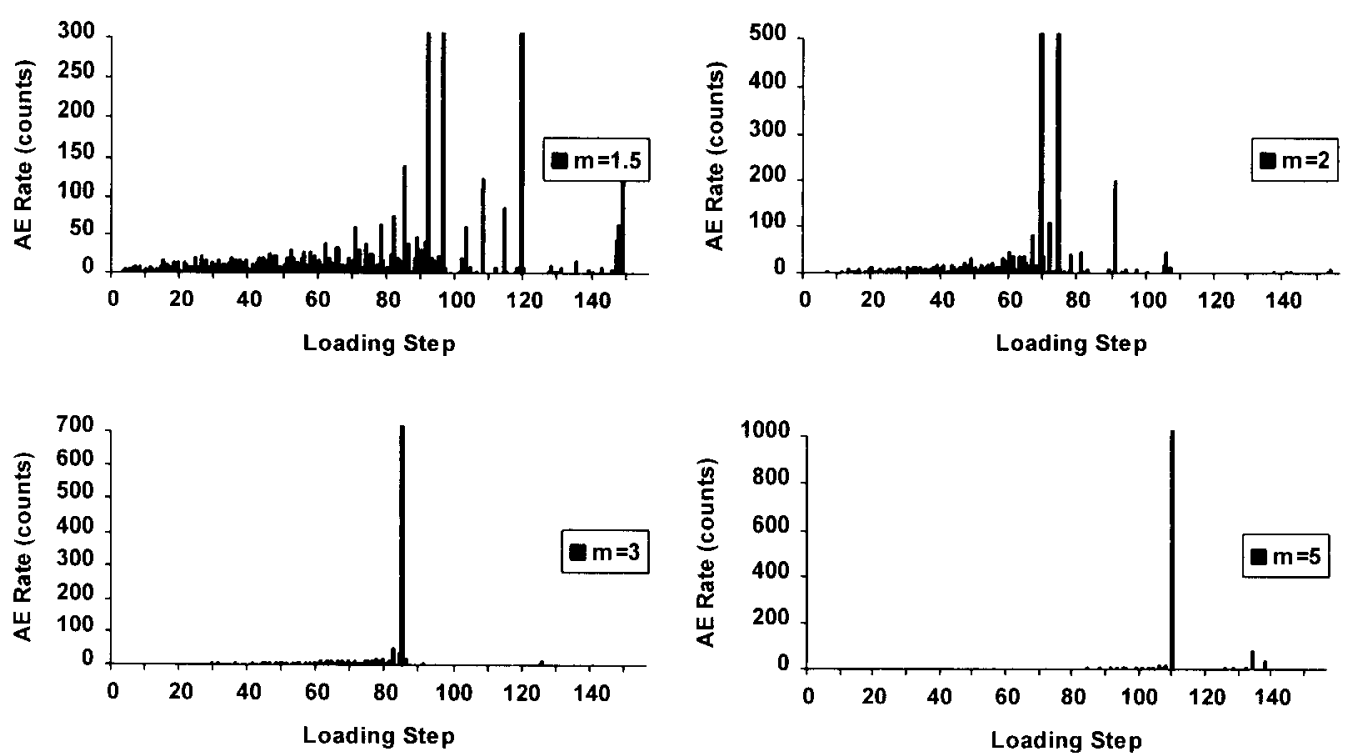

Fig.3 Influence of heterogeneity on AE patterns for specimens under compression

\subsection{Influence of Heterogeneity on Failure Modes}

One of the most important characteristics of post-peak behavior of brittle material under any loa ding scheme is the localization of deformation [2]. After reaching the maximum strength of the spec imen, the deformation starts to concentrate in the fracture process zone (FPZ), while other parts of $t$ he specimen unloaded (the so-called elastic rebound in geophysics). Fig.4 and Fig. 5 show that the $\mathrm{c}$ urrent numerical model is capable of capturing this phenomenon. Comparing the stress-strain curve shown in Fig. 1 or Fig. 2 and the failure processes shown in Fig.4 or Fig.5, it is found that, few distri buted fractures appear up to approximately $80 \%$ of the maximum strength. The number of these frac tures increases considerably as the load approaches its ultimate value. Beyond the ultimate load, the se fractures begin to concentrate in a certain zone. The maximum strength does not necessarily mea $\mathrm{n}$ an abrupt failure of the specimen. Although the loading bearing capacity drops dramatically durin $\mathrm{g}$ the post-peak part, this does not indicate the collapse of the specimen. Subsequently, a distinct tra nsition occurs and the loading capacity begins to decrease at a much slower rate until it reaches its $r$ esidual strength, which is about $10-20 \%$ of its maximum strength.

\subsection{Influence of Heterogeneity on Residual Strength}

In Okubo and Fukui' experimental study, one result of particular interest was obtained in uniaxi al tension testing: a large amount of residual strength remains in the post-failure region [8]. Peng co nducted uniaxial tension tests and also found considerable large residual strength for tested four roc ks [9]. The presence of residual strength in the post-peak region may be attributable to several reaso ns [9]. Heterogeneity or strength variation in a specimen is considered to be one of the reasons. If to cal strength variation is large, specimen failure starts in weak portions. Even in the post-peak regio n. strong portions are still intact and some amount of loading capacity remains whereas a macro-cra 
ck extends from boundary to boundary in a heterogeneous specimen.

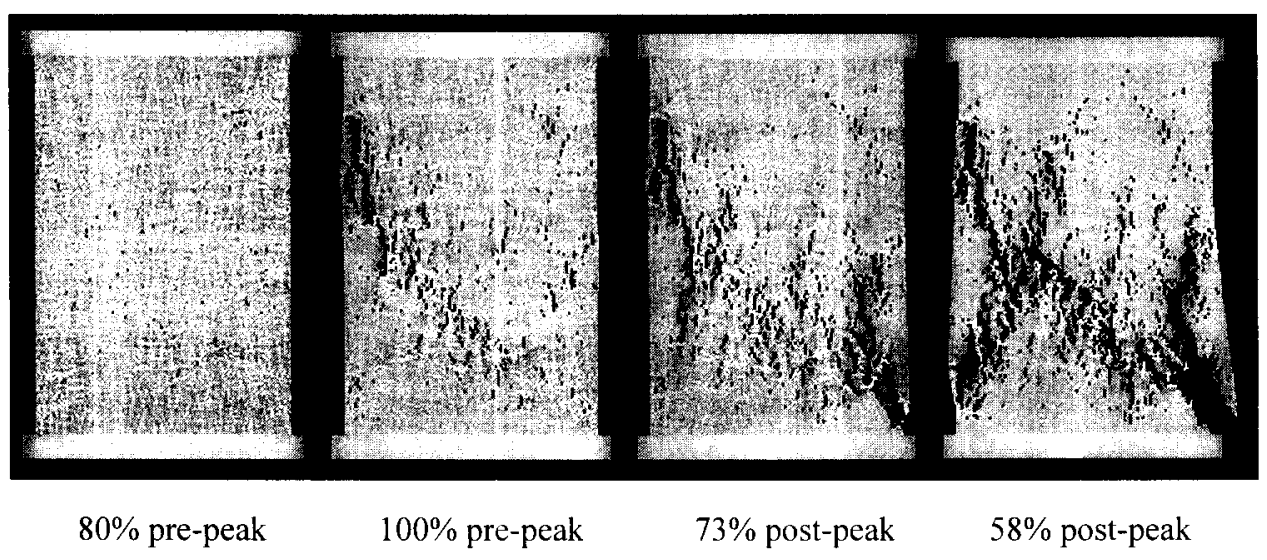

Fig.4 Numerical simulation of failure mode for specimen with $m=1.5$ under compression

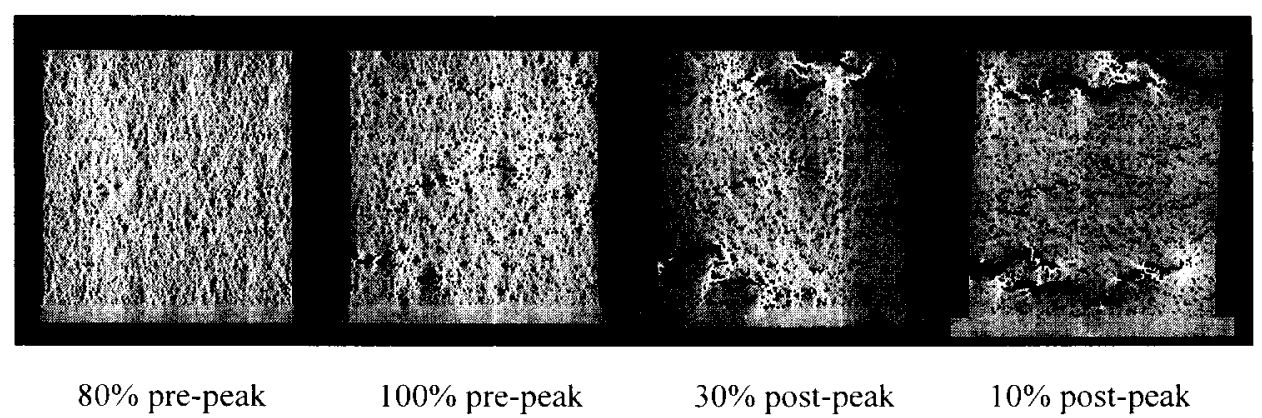

Fig. 5 Numerical simulation of failure mode for specimen with $m=1.5$ under tension

The above mentioned reason can be understood well by studying the final failure mode of speci men as shown in Fig.5. Four detailed figures of the development of the crack interface bridging in $t$ he specimen in tension are shown in Fig.5. It is seen that at the final loading step, the crack has alm ost cross the section of the specimen but still showed a non-zero residual stress and further loading (tensile deformation) is very difficult to drive the cracks for a visible propagation. Clearly the crack $\mathrm{s}$ are not continuous, but rather overlaps and branches exist. Note that these overlaps are not isolate $\mathrm{d}$ events in individual specimens, but they have been detected in relatively large quantity [10].

\section{CONCLUDING REMARKS}

It is important to identify the main failure mechanisms associated with brittle failure under compressive and tensile loading. This identification is crucial for better understanding and interpreting the experimental results and consequently, improves our concepts in material property design or analysis of rock or civil engineering structures. The model predications of brittle failure behavior of disordered materials in this paper capture most of the experimental observed 
phenomena, including softening, deformation localization, and crack patterns. Although the simulations are not a quantitative approach and many conclusions presented here may have already been obtained by laboratory tests, the significance of mimicking these phenomena by numerical simulation is obvious. At least, and the most important, the successful reproducing of the experimentally observed failure phenomena with a numerical method implies that our understanding to the mechanisms of material failure has reached a more reasonable level, which in turn will help us to make further progresses in the field of material mechanics and rock or civil engineering. Fig. 6 shows three examples of tentative application of MFPA ${ }^{2 D}$ in modeling failure of composite materials or rock engineering structures.

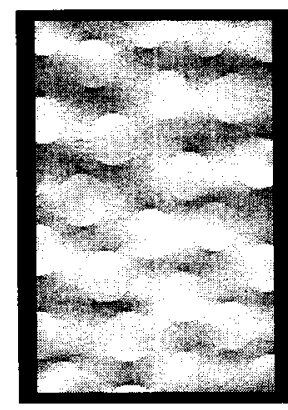

Particle composite

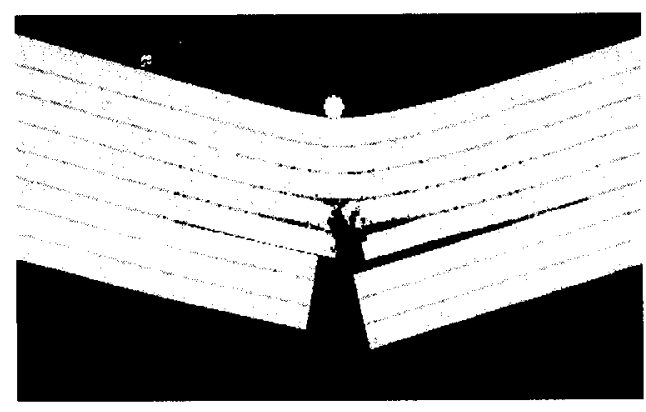

Failure process of interface material

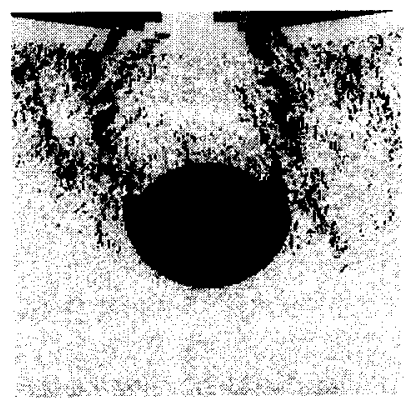

Tunnel failure

Fig.6 Examples of MFPA ${ }^{2 D}$ applications on modeling material failure or construction collapse

\section{ACKOWNLEDGMENT}

The authors are grateful for the support of this work from the Chinese National Key Fundamenta 1 Research "973 Programme" (No.95-13-07-01) and the National Natural Science Foundation (No. 49974009).

\section{REFERENCE}

1. C.A.Tang, Int. J. Rock Mech. Min. Sci., 34 (1997) p.249

2. A.R.Mohamed and W. Hansen. ACI Materials J., 96 (1999) p.196

3. S.C. Blair and N.G.W. Cook, Int. J. Rock Mech. Min. Sci., 35 (1998) p.837

4. J.G.M.Van Mier, Fracture process of concrete, CRC Press, New York (1997) p.253

5. P.A.Cundall., Int. J. Rock Mech. Min. Sci., 25 (1998) p. 107

6. R.Hart, P.A.Cundall and J.Lemos, Int. J. Rock Mech. Min. Sci., 25 (1988) p.117

7. S.J.D.Cox and L.Paterson, Deformation Mechanics, Rheology and Tectonics, Geological Societ y Special Publication No.54 (1990) p.57

8. S.Okubo and K.Fukui, J. Rock Mech. Min. Sci., 33 (1996) p.549

9. S.S.Peng, Int. J. Rock Mech. Min. Sci., 12 (1975) p.125.

10. Van Mier J.G.M, Fracture process of concrete, CRC Press, New York, 1997. 
Fracture and Strength of Solids IV

10.4028/www.scientific.net/KEM.183-187

On Failure Modes and Strength Characterization of Brittle Disordered Materials under Uniaxial Compression and Tension

10.4028/www.scientific.net/KEM.183-187.637

\section{DOI References}

[10] Van Mier J.G.M, Fracture process of concrete, CRC Press, New York, 1997. doi: $10.1177 / 105678959700600403$ 